\title{
Foot Digit 3
}

National Cancer Institute

\section{Source}

National Cancer Institute. Foot Digit 3. NCI Thesaurus. Code C52841.

The middle or third toe from the medial side of the foot. 